\title{
DEVICE FOR ASPIRATION OF OCULAR HUMOURS*
}

BY

\author{
ALMIRO AZEREDO \\ Faculty of Medicine of Ribeirão Prêteo, University of São Paulo, Brazil
}

THE purpose of the instrument here described is to aspirate ocular fluids for analyses (Figs 1 and 2). It was planned for the uveitis clinic at the University of São Paulo.

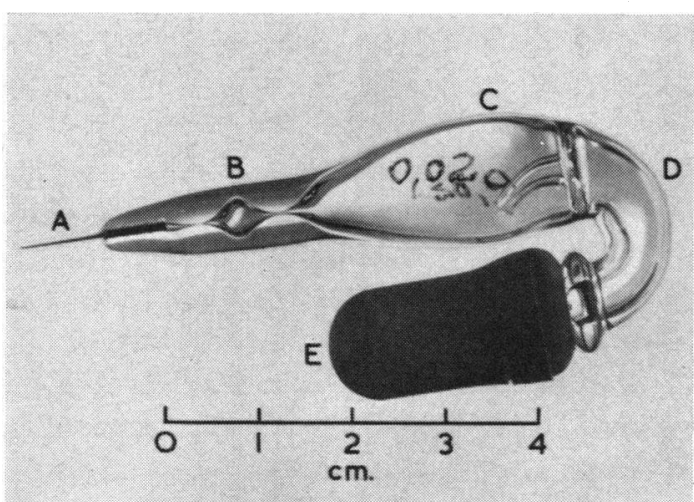

Fig. 1.-Instrument for aspiration of ocular humours.

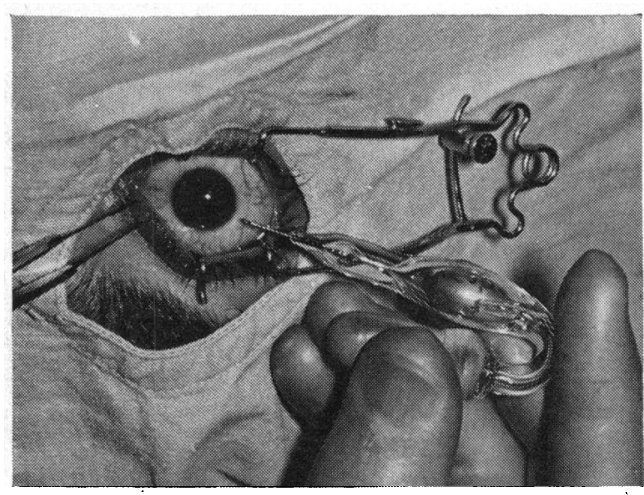

FIG. 2.-The instrument in use.

A regular stainless steel needle, $1 \mathrm{~cm}$. long, with a sharp and short bevelled point (A) perforates the cornea (or sclera) while the fingers press the rubber bulb (E) and close the vent (D) which connects the glass (pyrex) bulb (C) with the atmosphere. By gradually releasing that pressure, the ocular fluid under examination (aqueous or vitreous) fills the reservoir (B) when the vent is opened in order to avoid the reflux of the sample into the glass bulb. This fluid is kept in the reservoir until analysed and is expelled from it by pressing the rubber bulb and closing the vent at the same time.

The model for vitreous aspiration has a 10-gauge needle, the one for aqueous a 6-gauge needle. The capacity of the reservoir, also made of pyrex, may vary from 0.02 to $1 \mathrm{ml}$., according to the volume necessary for analyses.

The chief advantage of the instrument lies in its compactness. Only one hand is needed to manipulate it, making for greater precision in surgical operation, since the hand may rest on the patient's face.

Mr. J. C. Brandão, glass technician, made the instrument. 